\title{
Biodeterioration of Microplastics: A promising step towards Plastics Waste Management
}

\section{Aatikah Tareen ( $\square$ atikatarin@gmail.com )}

WUM: The Women University Multan

\section{Atia lqbal}

WUM: The Women University Multan

Nazia Jamil

University of the Punjab

Original article

Keywords: Micro-pollutants, Landfill, Soil biota, polyethylene, Polyester

Posted Date: May 13th, 2021

DOI: https://doi.org/10.21203/rs.3.rs-505761/v1

License: (1) This work is licensed under a Creative Commons Attribution 4.0 International License.

Read Full License 


\section{Abstract}

Polyethylene and Polyester materials are resistant to degradation and found as significant source of microplastics pollution, which is emerging concern. In the present study, potential of dumped site bacterial community was evaluated. After primary screening it was observed that $68.5 \%$ were linear lowdensity polyethylene, $33.3 \%$ were high-density and $12.9 \%$ were Polyester degraders. Up to five strains were chosen for secondary screening, where they were monitored by FTIR, SEM and weight loss degradation trials. Major results were observed by Alcaligenes faecalis (MK517568) and Bacillus cereus (MK517567), as they show highest degradation activity among others. Alcaligenes faecalis (MK517568) degrade LLDPE by $3.5 \%$, HDPE by $5.8 \%$ and Polyester by $17.3 \%$. Bacillus cereus (MK517567) better tolerated at $30^{\circ} \mathrm{C}$ and degrade Polyester by $29 \%$. Changes in infrared spectra indicated degradation pathways of different strains with types of plastics targeted. Through SEM analysis, groves, piths and holes were observed on surface. These findings suggest that soil bacteria develop effective mechanism for degradation of microplastics and beads that enables them to utilize plastics as source of energy without need of pre-treatments, which highlights importance of these soil bacteria for fate of effective plastic waste management in soil environment.

\section{Introduction}

After all the development over a hundred years back, plastics have been utilized in numerous approaches, and they are associated with each part of our lives (Zhang et al. 2019). Regardless of their benefits, issues identified with its wide applications can't be ignored. Most of these plastics are resistant and remain in earth for an inclusive generation (Tiwari et al. 2020). According to recent information, the worldwide plastic production reaches to 360 million tons in 2018. Asia is the biggest maker and consumer of world's plastics. Among worldwide plastic producers, China subsidizes the most elevated part (30\%) trailed by Europe (17\%) and totally $18 \%$ is from Canada, Mexico and US (Plastic Europe 2019). It is appropriate to specify that petrochemical plastics share over $80 \%$ of the overall plastic use. For instance, polyethylene terephthalate, Polyester, polyethylene, polypropylene, polystyrene, and polyvinyl chloride are the commonly utilized plastics (Urbanek et al. 2018). Various strategies utilized for plastic waste management are garbage lot, burning and reusing, every one of these techniques has its disadvantages, for example plastics are burnt or incorporated into landfills utilizes sources of land, which causes pollution and limited natural resources are being wasted. Currently limited quantities are reused and this causes prevention to the idea of circular economy (Chen et al. 2020).

Among the plastics which resides, fragmented particles have gained attention and are of more troubling. These scrapes are characterized as microplastics $(0.1 \mu \mathrm{m}-5 \mathrm{~mm})$ based on their diverse range (EerkesMedrano et al. 2015; Gigault et al. 2018). Due to smaller size and wide dispersion of these fragments, it is hard to gather them all-out and afterward their removal from environment. In the same way, the speed at which they enter in nature outstripped their clearance speed (Auta et al. 2017). Besides, microplastics found in potable water, which may become a danger to wellbeing (Novotna et al. 2019). Polyethylene is a generally utilized type of plastic because of it convey ability. Nonetheless, because of its saturated linear 
hydrocarbon chains, and can be communicated as - [CH2-CH2], PE items are hard to be corrupted by nature (Kumar Sen and Raut 2015). Feasible way-out to this issue is conversion of plastics by microbes. Microbes that have potential for degradation of plastics mostly belong to phyla Proteobacteria, Firmicutes and Actinobacteria, and most of them were screened from polluted dumpsites. The strategies given, by screening with electron microscopy and Fourier-transform infrared spectroscopy. Studies should concentrate on biodegradation of microplastics emerging from the most well-known contaminant such as polyethylene (Matjasic et al. 2020).

Microbial assisted deterioration of polymers by bacteria and fungi have been effectively researched from indigenous habitats, such as soil. For example, Bacillus sp., Rhodococcus sp. (Auta et al. 2018), Pseudomonas aeruginosa (Jeon and Kim 2015), Zalerion maritimum (Paço et al. 2017), and Aspergillus clavatus (Gajendiran et al. 2016) can utilize plastics polymers as a matter of their energy source in cultivation media, therefore causes decrease in dry weight of polymers and incite physical-chemical alterations which include modifications in surface morphologies. Subsequently these microbes forming cracks and rough surfaces and producing chemical bonding structures, such as carbonyl groups, ketones and aldehydes (Mukherjee et al. 2015). Microorganisms could break down the polymers in two phases, firstly taking inside them and then enzymatically dissimilated to discharge extracellular proteins, causes chain cleavage into monomer, which can be used by microorganisms (Auta et al. 2018). Microbial species have ability to change polymers into monomers and additionally dissimilate into carbon dioxide and water. Biodegradation accomplished by microbes is related with key characteristics, for instance, atomic weight and crystallinity of the polymers (Glaser 2019; Gewert et al. 2015; Nauendorf et al. 2016).

The aim of the present study is to evaluate potential of locally isolated strains to degrade plastics with different chemical compositions based on their petrochemical origin. Alcaligenes faecalis, Bacillus spp. and Staphylococcus sp. isolated from dumped areas of Multan with affinity for LLDPE, HDPE and Polyester with considerately faster decay rate, which is globally needed especially for developing countries.

\section{Material And Methods}

\section{Polyethylene and Polyester used in current study}

Linear low-density polyethylene, high-density polyethylene and polyester beads and plastics powders used in current study were provided by Mehran plastic industries Pvt Limited, Pakistan. LLDPE, HDPE and polyester have fine powders with $99.5 \%$ purity. Beads were sterilized, followed by washing with $70 \%$ ethanol and drying beads with filter paper and make them ready-to-use in experimentation.

\section{Isolation, Identification, and Screening of bacterial isolates}

Samples of soil (5g) were collected from different municipal dumpsites in Multan which are heavily dumped with various plastics waste along commercial areas. These specified areas were chosen since it was used as plastic dump site for a very long time, expanding likelihood of finding bacteria that has 
ability to degrade plastics. By using conventional serial dilution method, $50 \mu$ from $10^{-6}$ dilution was spread on nutrient agar (Sigma Aldrich) plate infused with 1\% of plastic powder for isolation of plastic degrading bacteria, and for each type of given plastic this procedure was repeated. After fifteen days of incubator at $37^{\circ} \mathrm{C}$, bacteria with distinct zones were selected and sub-cultured for further characterization (Auta et al. 2017). Based on morphological characterization of bacterial colony after primary screening, Gram-stained slides was observed under light microscope at 40X and 100X for identification.

Secondary screening of isolates performed by both static and shaking methods using mineral salt medium previously used by Osman et al (Osman et al. 2018) supplemented with $5 \%$ polyethylene (LLDPE and HDPE) and polyester powder. Isolation of plastic degrading bacteria was verified by streak the bacteria on $5 \%$ concentration solid media and diameter of zone was observed after 15 days of incubation at $37^{\circ} \mathrm{C}$, while shaking technique involve biodegradation assay and liquid media. After secondary screening, molecular identification performed by amplification of $16 \mathrm{~S}$ rRNA gene by using primers $785 \mathrm{~F} 5^{\prime}$ (GGA TTA GAT ACC CTG GTA) 3', 27F 5' (AGA GTT TGA TCM TGG CTC AG) 3 from Macrogen sequencing system. Sequences were analyzed utilizing BLAST tool of National Centre for Biotechnology Information (NCBI) (https://www.ncbi.nlm.nih.gov/) against reference 16S rRNA sequences of type strains and submitted to GenBank. To construct consensus neighbor joining tree phylogenetic analysis was carried out by using Molecular Evolutionary Genetics Analysis (MEGA) version 7 software (Ahmad et al. 2018).

\section{Rate of deterioration by weight loss strategy and the impact of temperature and $\mathrm{pH}$ on weight of plastic}

Isolates showing zone of clearance in static secondary screening were further assessed for the degradation study. Each isolate was separately inoculated to flasks containing of $20 \mathrm{ml}$ of minimal medium and beads of Polyethylene (LLDPE and HDPE) and Polyester separately. The biodegradation study was implemented for a time of 40 days. Analysis was done by weight reduction using following formula (Mohan et al. 2016).

$($ Weight loss $\%=$ initial weight - final weight / Initial weight $\times 100)$

Biodegradation study of LLDPE, HDPE and Polyester was performed at different temperature and $\mathrm{pH}$ conditions in order to optimize the isolated bacterial cultures in different environmental conditions, for future consequences. The range of temperature used in this study were $30^{\circ} \mathrm{C}$ and $45^{\circ} \mathrm{C}$, however, the pH was maintained at 4.0 and 8.0 scale. The weight of plastic beads was recorded earlier. Optimization of isolates were carried out for 15 days (Kale et al. 2015).

\section{Formulation of microbial consortia and determination of weight loss for mixture of plastic pellets in local natural conditions}

An acclimated consortium capable of degrading polyester, high-density and linear low-density polyethylene pellets developed in laboratory. A 500ml flask with nutrient broth (Sigma Aldrich) inoculated with Alcaligenes faecalis, Streptococcus sp. and three types of Bacillus spp., were operated for the degradation experiment. Subsequently, consortium was cultured until lag phase was developed for 6 
days. A control was set up containing just broth and beads of LLDPE, HDPE and polyester (Tsiota et al. 2018). To examine biodegradation of LLDPE, HDPE and Polyester in ex-situ condition. The biodegradation of commercially available plastic beads under the attack of bacteria was studied using the soil burial method for interval of three months. Pre-weighted beads were washed with $70 \%$ ethanol and added to soil. Sterilized pellets were added to soil along nutrient broth and inoculum. Soil selected was clay loam with $\mathrm{pH}$ 8.0. Experiment proceeds from March to May so temperature variations $\left(23^{\circ} \mathrm{C}\right.$ to $45^{\circ} \mathrm{C}$ ) were noted.

\section{Analysis of Plastic Beads Degradation by Sturm test}

Plastic degrading bacteria can break down the long chains of polymers into monomers by their different activities, either by oxidation or enzymatic hydrolysis. $\mathrm{CO}_{2}$ evolved as a result of mineralization of plastics in aerobic conditions. Evolution of $\mathrm{CO}_{2}$ by bacterial isolates can be assessed by sturm test applied in modified way. Pre-weighted beads of LLDPE, HDPE and polyester were added to flask containing $5 \mathrm{ml}$ of MSM broth and $1 \mathrm{ml}$ of $\mathrm{KOH}(1 \mathrm{M})$ was added. Inoculated with 24 hours old culture and incubated in rotatory incubator for 25 days. Aerobic conditions were maintained in rotatory incubator. After 25 days of incubation, the amount of carbon dioxide generated was calculated in the test and control. $\mathrm{CO}_{2}$ produced as a result of mineralization was trapped in flask containing $\mathrm{KOH}$. Barium chloride solution Bacl2 (1M) was added to flask contained $\mathrm{KOH}$ as a result barium carbonate precipitates formed. Precipitates were filtered on filter paper and dried them at $50^{\circ} \mathrm{C}$ in an oven for an hour. The weight of precipitates indicates the $\mathrm{CO}_{2}$ generation by test organism as their end product (Singh et al. 2014).

\section{Surface Modification Analysis of Plastic Beads by Scanning Electron Microscopy}

Degraded beads of aromatic polyester, linear-low density and high-density polyethylene were set up on aluminum-disk with width of $1.2 \mathrm{~cm}$ using double sided black colored carbon tape. Sputtering of samples with a thin layer of gold in a vacuum chamber was done by using argon gas and an electric current of approximately $3 \mathrm{~mA}$. Then labelled tests pellets were placed sequentially in scanning electron microscope chamber and magnification was set to display images at 500x, 1000x and 10,000x with a TESCAN MERA 3 field emission scanning electron microscope (Dharmalingam et al. 2015).

\section{Structural Changes of Plastic Beads Analysis By Fourier Transform infrared Spectroscopy}

The LLDPE, HDPE and Polyester beads treated with isolated bacteria for a time of 40 days were examined by FTIR. Background noise was eliminated by performing a blank scan in the frequency range of 4000 to $600 \mathrm{~cm}-1$. Thus, the samples were scanned in the region of $400-4000 \mathrm{~cm}^{-1}$ at a resolution of $4 \mathrm{~cm}^{-1}$. The resultant spectrum included a plot of rate transmittance versus wave number which was additionally investigated against respective comparative controls (Shakirchan et al. 2017).

\section{Results}


Isolation and screening of bacteria with potential to degrade microplastics of LLDPE, HDPE and Polyester

In present study isolation of effective polyethylene (LLDPE, HDPE) and aromatic polyester degrading bacteria from municipal landfill soil were carried out. The dump sites soil samples were collected from old historic Daulat gate of city, Northern by-pass, Sher-shah road and Shujabad road. Morphologically distinct isolates were used to determine its ability to use plastic as carbon source. The experiments were conducted over a screening series. Primary screening led to the isolation of 54 bacterial isolates. Out of 54 isolates, 37 considered as linear low-density polyethylene degraders (68.5\%), 18 isolates were highdensity polyethylene degraders (33.3\%) and 7 isolates were polyester microfibers degraders (12.9\%). Out of 54 isolates, bacterial isolates which show distinct growth and zone were streaked on $5 \%$ concentration of each given type of microplastics and observed by formation of a clear zones. Alcaligenes faecalis (SA5) shows zone of clearance with $3 \mathrm{~mm}$ for polyester microfibers and $1 \mathrm{~mm}$ for LLDPE. Bacillus cereus (SA68) shows $6 \mathrm{~mm}$ diameter for LLDPE and $2 \mathrm{~mm}$ for HDPE. Bacillus spp. (SB-14) shows $4 \mathrm{~mm}$ diameter for LLDPE and $0.25 \mathrm{~mm}$ for polyester microfibers, another Bacillus spp. (SC-9) shows $1.5 \mathrm{~mm}$ diameter for HDPE and $3 \mathrm{~mm}$ for LLDPE microplastics. Streptococcus spp. (SC-56) shows $5 \mathrm{~mm}$ zone diameter for LLDPE and $0.5 \mathrm{~mm}$ diameter for HDPE microplastics. These five isolates show prominent zones, and tested further for biodegradation assay.

\section{Assessment of the polyethylene (LLDPE and HDPE) and Polyester deteriorating bacteria based on weight loss percent in ex-situ and laboratory conditions}

The degradation was determined by calculating the percentage of weight loss in polyethylene and polyester beads by isolated bacteria after 40 days of incubation. Linear low-density polyethylene degraded by SA-5 (Alcaligenes faecalis) (MK517568), SA-68 (Bacillus cereus) (MK517567), Bacillus sp. (SB-14 and SC-9) and Streptococcus spp. (SC-56) by 3.5\%, 15\%, 11.8\%, 4.8\% and 9.8\%. High density polyethylene degraded by SA-5 (Alcaligenes faecalis) (MK517568) Bacillus sp. (SB-14 and SC-9) and Streptococcus spp. (SC-56) by $5.8 \%, 11.7 \%, 3.8 \%$ and $13.7 \%$. Polyester was degraded by SA-5 Alcaligenes faecalis (MK517568), Bacillus sp. (SB-14 and SC-9) by 17.3\%, 9.4\% and 5.8\%. Difference between initial and final weight indicate the extent of polyethylene and polyester utilization by the bacteria significantly as shown in figure no. 1. Replicates were used to gain statistical confidence as standard deviation used, while control (no bacteria) shows zero percentage of degradation and beads were floating in the media. In the treatment flasks beads were settled down due to bacterial action.

After biodegradation assay, bacteria were optimized. After 15 days of incubation with continuous shaking, maximum percent reduction in weight or percent loss in weight (\% WL) of the polyester beads was recorded with Bacillus cereus (MK517567) $(29.4 \pm 0.05)$ at $30^{\circ} \mathrm{C}$ which was recorded as highest weight loss during experiment. Alcaligenes faecalis (SA-5) revealed $6.6 \%$ polyester-degradation at $45^{\circ} \mathrm{C}$. Polyester degradation was carried out at $30^{\circ} \mathrm{C}$ as well as at $45^{\circ} \mathrm{C}$ by both isolates (SA- 5 and SA-68) respectively. In addition to this, $3.5 \%$ high density polyethylene degradation was recorded by Alcaligenes faecalis (SA-5), Bacillus cereus (SA-68) gives $(6.6 \pm 0.02)$ for $\mathrm{HDPE}$. At $30^{\circ} \mathrm{C}$ the highest weight loss

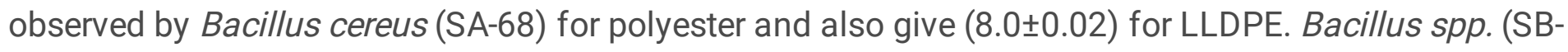


14 and SC-9) and Streptococcus sp. not shows much promising results in changing environmental conditions. SA-5 and SA-68 were considered as promising degraders and were identified by sequencing.

The percentage of weight loss in natural condition found to be $4.3 \pm 0.02,4.8 \pm 0.02$ and $2.7 \pm 0.02$ for LLDPE, HDPE and polyester beads after 90 days of incubation, respectively. The weight loss percentage of plastics strips were found to be $4.8 \pm 0.02$ and $4.9 \pm 0.02$ for LLDPE and HDPE after 90 days of incubation, respectively. In the end of incubation, it was analyzed that plastic strips are easy targets for bacteria as compared to beads and the possible reason for this may be bacterial strain utilized the released $\mathrm{C}$ compounds from plastic strips during the degradation. It is suggested that the result with this type of method was observed for first time.

\section{Characterization and Molecular Identification}

The morphological characteristics of the bacterial isolates were identified by conventional methods. Gram staining of bacterial isolates open up that they are Gram-positive rods and cocci. Growth on nutrient media with small, circular, flat, white colored, opaque or translucent colonies with smooth edges were observed on the plates. Biochemical testing revealed that isolates are catalase, indole and MRVP negative while give positive for starch hydrolysis. SB-14 gives yellow butt and red slant with gas production. Based on the 16S rRNA gene sequencing and biochemical characterization, the bacteria isolated from the site mainly belonged to Bacilli in terms of Bacillus cereus and Alcaligenes. As inferred by 16 S rRNA gene analysis, strain SA-68, showed $99 \%$ similarity with corresponding gene sequences of reference strains Bacillus cereus SBMWI and strain SA -5 showed $100 \%$ similarity with Alcaligenes sp. (KX164437.1) respectively. Phylogenetic trees (Figure no. 2 and 3) showed that strain was clustered on separate branches with reference strains belonging to respective genera. The GenBank nucleotide accession numbers assigned to strain Bacillus cereus SA-68 is MK517567 respectively. Query sequence designated as Alcaligenes faecalis with GenBank accession no. MK517568.

\section{Degradation of Plastic Beads Confirmed by $\mathrm{CO}_{2}$ Production in Sturm Test}

Carbon dioxide evolved as a result of deterioration of polyethylene (LLDPE, HDPE) and polyester by Alcaligenes faecalis (SA-5), Bacillus cereus (SA-68), Bacillus spp. (SB-14 and SC-9) Streptococcus sp. (SC-56) was trapped and compared to amount evolved in case of biotic control under similar conditions. Evolved carbon dioxide calculated from media inoculated was $0.602 \mathrm{~g}, 0.427,0.723,0.524$ and $0.205 \mathrm{~g} / 5 \mathrm{ml} \mathrm{CO} 2 \mathrm{~g}-1$ of $\mathrm{C}$, respectively. While the control flask indicates no precipitates.

\section{Surface Modifications Confirmed by Scanning electron microscopic analysis}

In this study, properties were authenticated with SEM and FTIR analysis. Degradation and morphological changes on polyester, liner-low density and high-density polyethylene pellets after bacterial treatment were analyzed by scanning electron microscopy. At length of all samples, degradation was observed due to roughness of surfaces and formation of cracks/holes/scions. (Figure 4, 5,6). Bacterial cells attached to surfaces were also visualized on some tested pellets. Scanning electron micrographs revealed that 
isolates cause localized surface deterioration over the plastics pellets, while the outside of untreated plastics is flawless and smooth even following 40 days of incubation. LLDPE samples surface indicates bacterial attachment and as well as bacterial actions on surface and study explain it at 500x, while HDPE and polyester explain at 1000 and 10,000x for better understanding.

\section{Fourier-transform infrared spectroscopy (FTIR) analysis}

The changes in spectral peaks due to biodegradation were determined using FTIR (ATR-alpha Bruker) spectrophotometer. The degradation of linear-low density and high-density polyethylene and aromatic polyester was confirmed with changes in spectra of FTIR analysis. The pellets without any treatment served as control, and pellets of LLDPE, HDPE and Polyester treated with isolated Alcaligenes faecalis, Bacillus sp. and Streptococcus spp. in MSM broth act as test. In case of LLDPE, the results of this study demonstrated that LLDPE pellets shown several peaks in the range from 2900 to $715 \mathrm{~cm}-1$, the vibrational mood of peaks were observed in comparison with control, the first peak at 2913.92 (control) shifts to $2913.83 \mathrm{~cm}-1$ which indicates the $\mathrm{C}-\mathrm{H}$ stretching of methyl group, the second peak at 2846.83 (control) shifts to $2846.50 \mathrm{~cm}-1$ which indicates the $\mathrm{C}-\mathrm{H}$ symmetric and asymmetric stretching of methylene ( $\mathrm{C}-\mathrm{H} 2)$, the third peak at 1462.50 (control) shifts to $1462.05 \mathrm{~cm}-1$ which indicates the $\mathrm{C}=\mathrm{C}$ and replacement of carbonyl bond with amine bond and the fourth peak at 718.05 (control) shifts to $718.23 \mathrm{~cm}-1$ which indicates the $-\mathrm{C}=\mathrm{C}$ - stretching and the presence of alkene group as shown in figure 7 indicate LLDPE and HDPE. For HDPE, the spectral peaks were observed from 2900 to $715 \mathrm{~cm}-1$ treated with bacterial isolates as shown in supplementary file. The four peaks were observed from control of HDPE at 2913.94 (C-H stretching - $\mathrm{CH} 3$ ), 2846.58(CH stretching - $\mathrm{CH} 2), 1461.42$ (bending C-H bond of methylene) and $718.23 \mathrm{~cm}-1$ (C-0). In case of treatments with bacterial isolates, the wave number of spectral peaks shown shifts from 2913.94(control) to $2914.37 \mathrm{~cm}-1$ which indicates the vibrations in stretching of $\mathrm{C}=\mathrm{C}$ bond seen in alkanes, second peak shown shifts from 2846.58 (control) to $2846.70 \mathrm{~cm}-1$ which indicates the stretching of $\mathrm{C}-\mathrm{H}$ bonds in methylene, the absorbance range of 3000 $2800 \mathrm{~cm}-1$ correspond to $\mathrm{C}-\mathrm{H}$ stretching and presence of alkanes. The third peak at 1461.42 (control) shown shift to $1461.76 \mathrm{~cm}-1$ which indicates the $-\mathrm{CH} 2$ stretching and presence of aromatics and the band around it also correspond to bending deformation. The fourth peak at $718.23 \mathrm{~cm}-1$ (control) shifts to $717.82 \mathrm{~cm}-1$ which indicates the rocking deformation of bonds. For Polyester, two treatments were given SA-5 and SC- 9 as both were showed the biodegradation for polyester among other treatments. The nine spectral peaks were observed in control of polyester at 2024.83(C-H), 1709.16(Carbonyl group), 1409.10(C=C), 1339.65(CH3), 1241.02(Ar-O-R), 1097.75(C-O), 1017.93(C-0), 871.00(Aromatic ring) and $720.01 \mathrm{~cm}-1$ (Mono-substituted aromatic ring). In treatment vibrational shifts were observed from 1709.16 (control) to $1710.84 \mathrm{~cm}-1$ which indicates a change in carbonyl group in polyester components and thus the cleavage of ester bond. Other peak from 1409.10 (control) shifts to $1408.45 \mathrm{~cm}-1$ which indicates the decrease in intensity of band leads to bond cleavage of $\mathrm{C}=\mathrm{C}$. The band from 1339.65 (control) shifts to 1340.04 which indicatesthe $\mathrm{CH} 3$ symmetrical bending. The peak from 1241.02(control) shifts to 1238.19 which indicates the assymetrical bend of Ar-O-R. The peak from 1097.75(control) shifts to1088.58 which indicates the bond cleavage of C-0, 1017.93(control) shifts to 1016.01 which indicates the bond cleavage of C-0. The peak 871.00 (control) shifts to 870.14 which 
indicates aromatic ring bend out of plane and the peak 720.01 (control) shifts to 719.4 which indicates the stretch of monosubstituted ring. The first peak was disappeared in treatment with SA-5 indicates that cleavage of $\mathrm{C}-\mathrm{H}$ bond was occurred which indicates the formation of new intermediate products as shown in supplementary file.

\section{Discussion}

Detrimental effects of plastic waste are increasing gradually. Therefore, its elimination from the earth is fundamental. Among thermic, photooxidative, mechanochemical, and catalytic degradation strategies, biodegradation is considered as the best choice for plastic waste degradation on account of minimal effort and eco-accommodating nature (Yogalakshmi and Singh 2020). But detailed characterization of proficient plastic-detiorating microorganisms and microbial compounds should be completed (Singh and Rawat 2020). Different studies demonstrate biodegradation of plastics by marine water isolates, rhizosphere samples, but this study isolate bacteria from municipal landfill sites, as these sites are capable to accommodate bacteria with high potential to degrade plastics and remain stable. The current study from Multan, Pakistan isolates fifty-four bacterial isolates with a potential to degrade different types of microplastics and beads and to best of authors knowledge no previous studies have been found for isolation of these bacteria from this region. So, these indigenously isolated strains can be valuable input for combating plastic pollution waste. Earlier in 2013 from Islamabad, Pakistan, Shah reported polyester-polyurethane degrading bacteria (Shah et al. 2013). Primary screening results in growth of isolates along with zones on infused media with plastics powder and repeated screening with $5 \%$ concentration leads to formation of clear zones ranges in 0.25 to $6 \mathrm{~mm}$. As compared to this, study conducted from Iraq the zones range from 2.5-3.0mm on 0.1\% LDPE infused MSM and implied that the bacterial colonies engendering a clear zone were capable of degrading polyethylene in minimal media (Auta et al. 2017). In the treatment flasks beads were settled down due to bacterial action.Many studies describe that film-type plastics required at least two months for biodegradation to occur (Yang et al. 2015) which is a very long time for testing and makes it unsuitable for identification of new bacterial strains. Alternatively, surface area of beads is larger than film of plastics, and chance of bacterial attachment increases which may speed up the reaction (Li et al. 2020) To test this, we prescribed 40 days of incubation to beads in liquid media, rate of biodegradation and efficiency were improved. The apparent degradation efficiency was assessed by weight loss of beads in MSM, and Bacillus cereus degrade LLDPE by $15 \%$ and Alcaligenes faecalis degrade polyester by $17.3 \%$ and weight loss for other types of plastic were also noted as mentioned in section. Weight reduction may have been due to different metabolism rate of different species. Different studies reported that rate of degradation of polyethylene varied from 1.5 to $13 \%$ as according to different assays and types of microbes (Park and Kim 2019). Another study from India also supports that Bacillus spp. are also plastic degraders by using Bacillus amyloliquefaciens as LDPE degraders (Das and Kumar 2015). All these studies indicate that these are degraders for specific type of plastic but for this study, Alcaligenes faecalis and Bacillus cereus have potential to degrade more than single type of plastic. Weight reduction was also optimized at different environmental conditions in laboratory and outside, in which Bacillus cereus (SA-68) show maximum 
tolerance and degrade polyester by $29 \%$ weigh loss after 15 days of incubation. In another study, optimization of growth media was carried in which $\mathrm{pH}$ of the media containing HDPE gradually decreases after 90 days of incubation from 7.3 to $5 \%$ (Ojha et al. 2017). But we optimized bacteria for their growth and potential ability to degrade plastic in different environmental conditions. Although different studies use Alcaligenes faecalis, Bacillus spp. and Streptomyces spp. as potential degraders. For another study, Alcaligenes faecalis was isolated from PET-treated soil and confirm as plastic degrader by FTIR analysis (Markandan et al. 2020). Another study from Iraq, uses Streptomyces spp. as source for pollution control but suggested best for LDPE degrader isolated from soil (Soud 2019). Above these, the effectiveness of degradation notice in this study was comparable to that of LDPE film degraded by mixed microbial cultures of Bacillus sp. and Paenibacillus sp. in which both bacteria together exhibit 15\% highest degradation efficiency (Das and Kumar 2015). As studies describe that plastics can be degraded into monomers, and then these monomers are converted into $\mathrm{CO}_{2}$ and water. To test this, sturm test was performed with a modified procedure. In another study the process used involves titration with thiosulphate and sodium hydroxide in the presence of barium chloride and $\mathrm{KOH}$. The highest rate of evolution for LDPE was $6.28 \mathrm{~g} /$ litre and lowest with $1.19 \mathrm{~g} /$ litre (Singh et al. 2014) as compared to this, the present study involves the $0.723 / 5 \mathrm{ml}$ noted. Curiously, the surfaces of pellets were seen to have become uneven and filled with cracks and groove (Auta et al. 2017). After thorough observations in different studies, they demonstrated that microorganisms can just change not only appearance but functional groups and other characteristics (Jeon and Kim 2013). In this study, these properties were authenticated with SEM and FTIR analysis. Previous studies utilized SEM micrographs as analytical tool to demonstrate erosions, cavities, and pores formed on plastic films to indicate the extent of colonization and degradation (Sowmya et al., 2014). Curiously, authors describe the surfaces of pellets were seen to have become uneven and filled with cracks and groove (Auta et al. 2017). Other studies also confirmed the degradation by formation of biofilm on plastic surface. From the study conducted in 2014 on LDPE degradation by Bacillus amyloliquefaciens isolated from municipal solid, SEM analysis revealed that both the strains were exhibiting adherence and growth with LDPE which used as a sole carbon source (Das and Kumar 2015). From another study that used photocatalytic technique for deterioration of polystyrene also indicates that cavities/holes were increase in size after the treatment (Sarwan et al. 2020). In present study it was also observed that cavities or holes produced in pellets. SEM analysis revealed surface erosion and bacterial adhesion by giving proof for the deterioration of the plastic beads because of activity of the bacteria and ensure the degradation capability of the bacteria. In addition to all these analysis, isolated strains efficiently degrade beads and microplastics of various categories of plastics. Hence, difference in biodegradation rate between LLDPE, HDPE and polyester may be due to presence of specific enzymes or concentrations of different enzymes required for different plastic degradation. Recommending that during growth of bacteria, may have different metabolism rate and uptake of energy from plastic as a source of carbon ( $\mathrm{Li}$ et al. 2020). PE microplastics can be categorized into HDPE and LDPE. Enzyme based degradation divided into two steps, extracellularly and intracellularly. In first step, LDPE broken down into shorter chains and next step followed by mineralization into $\mathrm{CO}_{2}$, $\mathrm{H}_{2} \mathrm{O}$ as described in sturm test. Laccase and Alkane showed reaction towards polyethylene belong to AlkB family enzymes, while Laccase is most reported enzyme responsible for HDPE degradation and 
alkane hydrolase for LDPE degradation (Ghatge et al. 2020, Montazer et al. 2020, Matjašič et al. 2021). Other studies also confirmed that manganese peroxidase and laccase enzymes produced by Bacillus cereus involved in degradation of low-density polyethylene after incubation of nine weeks followed by confirmation by FTIR (Sowmya et al. 2014). Another study explained that Alcaligenes faecalis produce extracellular enzymes like CMCase, protease, xylanase and lipase, which indicate that surface of plastic was attacked by thses enzymes followed by SEM and FTIR analysis (Nag et al. 2021). Montazer et al. explain that thses secreted bacterial enzymes followed formation of monomers by $\beta$-oxidation system pathway (Montazer et al. 2020). Spectral changes indicate the changes in formation of new bands at $1460,600-700 \mathrm{~cm}-1$. Our observations are similar with previous report in which bands at 2900, 720, 1460 cm-1 indicates the rocking deformation and stretching of bonds (Immanuel et al. 2017). In another study it was observed through FTIR that addition of -OH group to LDPE backbone was due to activity of bacteria producing enzymes for degradation of plastic (Roy et al. 2021). For this study, observations were made that indicates that cleavage in carbonyl group also suggest plastic degradation. In addition to all these analysis, isolated strains efficiently degrade beads and microplastics of various categories of plastics.

\section{List Of Abbrevations}

LLDPE (Linear low-density polyethylene)

HDPE (High-density polyethylene)

SEM (Scanning Electron Microscope)

FTIR (Fourier Transform Infrared Spectroscopy)

\section{Declarations}

\section{Ethics approval and consent to participate}

Not applicable

\section{Consent for publication}

All authors gave their informed consent prior to their inclusion in the study.

\section{Availability of data and materials}

Not Applicable

\section{Competing interests}

The authors declare that they have no competing interests. 


\section{Funding}

No funding was received.

\section{Authors' contributions}

Aatikah Tareen did data analysis, Aatikah Tareen and Atia Iqbal plan the research and check the manuscript, Nazia Jamil proof read the manuscript

\section{Acknowledgements}

Authors acknowledged by department of Microbiology and Molecular Genetics, The Women University, Multan.

\section{References}

Ahmad, F, Anwar, S, Firdous, S, Da-Chuan, Y, and Iqbal, S (2018) Biodegradation of bispyribac sodium by a novel bacterial consortium BDAM: Optimization of degradation conditions using response surface methodology. J. Hazard. Mater 349: 272-281.

Auta, H. S, Emenike, C. U, and Fauziah, S. H (2017) Screening of Bacillus strains isolated from mangrove ecosystems in Peninsular Malaysia for microplastic degradation. Environ Poll. 231: 1552-1559.

Auta, H.S, Emenike, C.U, Jayanthi, B, Fauziah, S.H (2018) Growth kinetics and biodeterioration of polypropylene microplastics by Bacillus sp. and Rhodococcus sp. isolated from mangrove sediment. Mar. Poll. Bull. 127:15-21.

Chen, Y, Awasthi, A. K., Wei, F, Tan, Q., Li, J (2020) Single-use plastics: Production, usage, disposal, and adverse impacts. Sci of Total Envir 141772.

Danso, D, Chow, J, Streit, W.R, (2019) Plastics: environmental and biotechnological perspectives Das, M.P, Kumar, S, (2015) An approach to low-density polyethylene biodegradation by Bacillus amyloliquefaciens. 3 Biotech 5:81-86.

Dharmalingam, S, Hayes, D. G, Wadsworth, L. C, Dunlap, R. N., DeBruyn, J. M, Lee, J, and Wszelaki, A. L (2015) Soil degradation of polylactic acid/polyhydroxyalkanoate-based nonwoven mulches. J. Polym \& Environ 23:302-315.

Eerkes-Medrano, D, Thompson, R.C, Aldridge, D.C (2015) Microplastics in freshwater systems: a review of the emerging threats, identification of knowledge gaps and prioritisation of research needs. Water Res 75:63-82.

Gajendiran, A, Krishnamoorthy, S, Abraham, J (2016) Microbial degradation of low-density polyethylene (LDPE) by Aspergillus clavatus strain JASK1 isolated from landfill soil. 3 Biotech 6:52-57. 
Gewert, B, Plassmann, M. M, and MacLeod, M (2015) Pathways for degradation of plastic polymers floating in the marine environment. Env Sci: Processes \& Impacts 17:1513-1521.

Ghatge S, Yang Y, Ahn J-H, Hur H-G (2020) Biodegradation of polyethylene: a brief review. Appl Biol Chem 63:27.

Gigault, J, Ter Halle, A, Baudrimont, M, Pascal, P.-Y, Gauffre, F, Phi, T.-L., El Hadri, H., Grassl, B., Reynaud, S (2018). Current opinion: What is a nanoplastic? Environ. Poll 518 235:1030-1034.

Glaser, J. A. (2019) Biological Degradation of Polymers in the Environment. In Plastics in the Environment. IntechOpen

Immanuel O. M, Ibiene A. A, and Stanley, H. O (2017) Enhanced biodegradation of polyethylene by fungus isolated from the koluama mangrove swamp in the Niger Delta. J. Microbial Biotech Res 4:1-9.

Jeon, H.J, Kim, M.N (2013) Biodegradation of poly(l-lactide) (PLA) exposed to UV irradiation by a mesophilic bacterium. Int. Biodeterior. Biodegrad 85:289-293.

Jeon, H.J, Kim, M.N (2015) Functional analysis of alkane hydroxylase system derived from Pseudomonas aeruginosa E7 for low molecular weight polyethylene biodegradation. Int. Biodeterior. Biodegrad. 103:141-146.

Kale, S. K, Deshmukh, A. G, Dudhare, M. S., \& Patil, V. B (2015) Microbial degradation of plastic: a review. J. Biochem. Technol 6: 952-961.

Kumar Sen S, Raut S (2015) Microbial degradation of low density polyethylene (LDPE): A review. J. Environ. Chem. Eng 3:462-473.

Li, J, Kim, H. R, Lee, H. M, Yu, H. C, Jeon, E., Lee, S, Kim, D. H (2020) Rapid biodegradation of polyphenylene sulfide plastic beads by Pseudomonas sp. Sci of Total Enviro, 137616.

Markandan, M., Sepperumal, U., Rodríguez, L. V. C. Bacterial (Alcaligenes Faecalis) Degradation of PET (Poly (Ethylene Terephthalate) Obtained from Old Bottles Wastes (2020).

Matjašič T, Simčič T, Medvešček N (2021) Critical evaluation of biodegradation studies on synthetic plastics through a systematic literature review. Sci Total Environ 752:141959

Matjašič, T, Simčič, T, Medvešček, N, Bajt, O., Dreo, T, \& Mori, N (2020) Critical evaluation of biodegradation studies on synthetic plastics through a systematic literature review. Sci of Total Enviro, 141959.

Mohan, A.J, Sekhar, V.C, Bhaskar, T, Nampoothiri, K.M (2016). Microbial assisted high impact polystyrene (HIPS) degradation. Bioresource Tech 213:204-207. 
Montazer Z, Habibi Najafi MB, Levin DB (2020) Challenges with verifying microbial degradation of polyethylene. Polymers (Basel) 12:123.

Mukherjee, S, Chowdhuri, U.R, Kundu, P.P (2015) Bio-degradation of polyethylene waste by simultaneous use of two bacteria: Bacillus licheniformis for production of bio-surfactant and Lysinibacillus fusiformis for bio-degradation. RSC Adv 6:2982-2992.

Nag, M., Lahiri, D., Dutta, B., Jadav, G., Ray, R. R. (2021). Biodegradation of used polyethylene bags by a new marine strain of Alcaligenes faecalis LNDR-1. Env Sci. Pol Res :1-15.

Nauendorf, A, Krause, S, Bigalke, N. K, Gorb, E. V., Gorb, S. N, Haeckel, M., and Treude, T (2016) Microbial colonization and degradation of polyethylene and biodegradable plastic bags in temperate fine-grained organic-rich marine sediments. Mar. Pollut. Bull. 103:168-178.

Novotna K, Cermakova L, Pivokonska L, Cajthaml T, Pivokonsky M (2019) Microplastics in drinking water treatment - Current knowledge and research needs. Sci. Total. Environ. 667:730-740.

Ojha, N, Pradhan, N, Singh, S, Barla, A., Shrivastava, A, Khatua, P., and Bose, S. (2017) Evaluation of HDPE and LDPE degradation by fungus, implemented by statistical optimization. Scientific Reports. 7(1): 1-13.

Osman, M, Satti, S. M., Luqman, A, Hasan, F, Shah, Z, and Shah, A. A (2018) Degradation of Polyester Polyurethane by Aspergillus sp. Strain S45 Isolated from Soil. J. Poly \& Env 26:301-310.

Paço A, Duarte K, Jo ao P, Santos, P.S.M, Pereira, R., Pereira, M.E., (2017) Biodegradation of polyethylene microplastics by the marine fungus Zalerion maritimum. Sci. Total Environ. 586:10-15.

Park, S. Y, Kim, C. G (2019) Biodegradation of micro-polyethylene particles by bacterial colonization of a mixed microbial consortium isolated from a landfill site. Chemosphere 222:527-533.

Roy, R., Mukherjee, G., Gupta, A. D., Tribedi, P., Sil, A. K. (2021) Isolation of a soil bacterium for remediation of polyurethane and low-density polyethylene: a promising tool towards sustainable cleanup of the environment. 3 Biotech. 11(1):1-14.

Sarwan, B., Acharya, A. D., Kaur, S., Pare, B. (2020) Visible light photocatalytic deterioration of polystyrene plastic using supported BiOCl nanoflower and nanodisk. Eur Poly J. 134: 109793.

Shah, Z, Krumholz, L, Aktas, D. F, Hasan, F, Khattak, M, and Shah, A. A (2013) Degradation of polyester polyurethane by a newly isolated soil bacterium, Bacillus subtilis strain MZA-75. Biodegradation 24:865877.

Singh, J, and Gupta, K. C (2014) Screening and identification of low-density polyethylene (LDPE) degrading soil fungi isolated from polythene polluted sites around Gwalior City (MP). Intern J Cur Micro App Sci 3:443-448 
Singh, S, Rawat, P. S (2020) Biodegradation of Plastic: An Innovative Solution to Safe the Human Health and Environment. In Handbook of Research on Environmental and Human Health Impacts of Plastic Pollution :435-461.

Skariyachan, S, Patil, A. A, Shankar, A, Manjunath, M, Bachappanavar, N, and Kiran, S (2018) Enhanced polymer degradation of polyethylene and polypropylene by novel thermophilic consortia of Brevibacillus sps. and Aneurinibacillus sp. screened from waste management landfills and sewage treatment plants. Polym Degrad and Stab 149:52-68.

Soud, S. A. (2019) Biodegradation of Polyethylene LDPE plastic waste using Locally Isolated Streptomyces sp. J. of Pharma Sci and Research. 11(4):1333-1339.

Sowmya, H. V, Ramalingappa, M. K, and Thippeswamy, B (2014) Biodegradation of polyethylene by Bacillus cereus. Adv in Polym Sci and Tech: Inter. J. 4:28-32.

Sowmya, H. V., Ramalingappa, M. K., and Thippeswamy, B. (2014) Biodegradation of polyethylene by Bacillus cereus Adv in Pol Sci and Tech: An Inter J. 4:28-32

Tiwari, N, Santhiya, D, Sharma, J. G. (2020) Microbial remediation of micro-nano plastics: Current knowledge and future trends. Environmental Pollution, 115044.

Tsiota, P, Karkanorachaki, K, Syranidou, E, Franchini, M, and Kalogerakis, N (2018) Microbial Degradation of HDPE Secondary Microplastics: Preliminary Results. In Proceedings of the International Conference on Microplastic Pollution in the Mediterranean Sea. 181-188.

Urbanek, A.K, Rymowicz, W, Mironczuk, A.M (2018) Degradation of plastics and plasticdegrading bacteria in cold marine habitats. Appl Microbiol Biotech 102:7669-7678.

Yang, Y, Yang, J, Wu,W.M, Zhao, J, Song, Y, Gao, L, Yang, R, Jiang, L, (2015) Biodegradation and mineralization of polystyrene by plastic-eating mealworms: part 2. Role of gut microorganisms. Environ Sci Technol 49:12087-12093.

Yogalakshmi, K. N, and Singh, S (2020) Plastic Waste: Environmental Hazards, Its Biodegradation, and Challenges. In Bioremediation of Industrial Waste for Environmental Safety. 99-133. Springer, Singapore.

Zhang, J.Q, Gao, D, Li, Q.H, Zhao, Y.X, Li, L, Lin, H.F, Bi, Q.R, Zhao, Y.C (2019) Biodegradation of polyethylene microplastic particles by the fungus Aspergillus flavus from the guts of wax moth Galleria mellonella. J. Sci. Total Environ. 704:1-29.

\section{Figures}




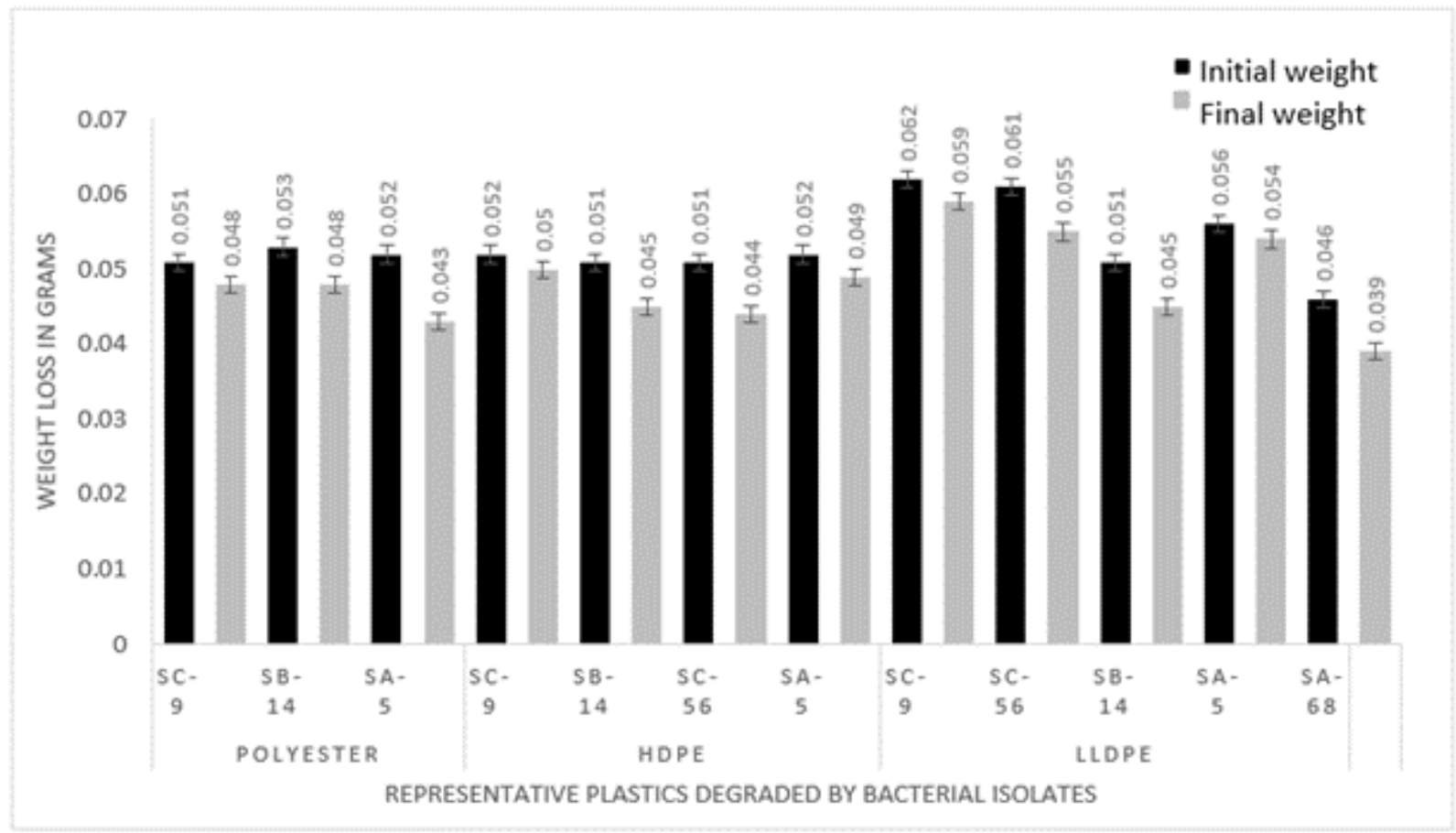

\section{Figure 1}

Significant weight reduction in plastic by bacteria-result shown by potential isolates Determination of weight loss in local natural conditions by consortium

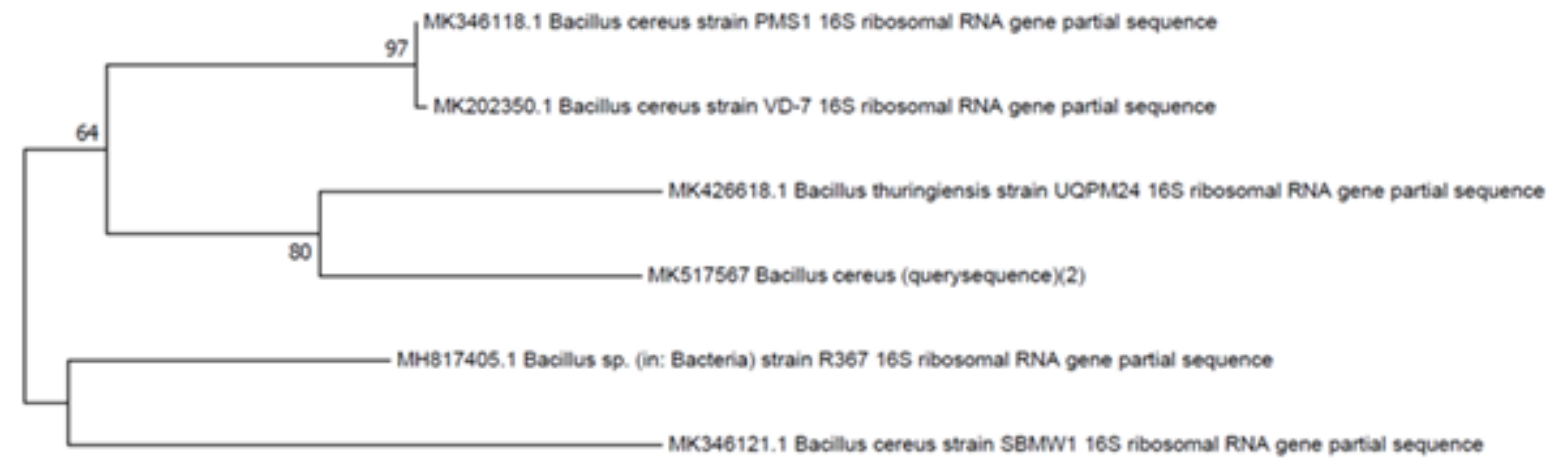

$\longmapsto$

Figure 2

Neighbor joining tree of Bacillus cereus (MK517567) 

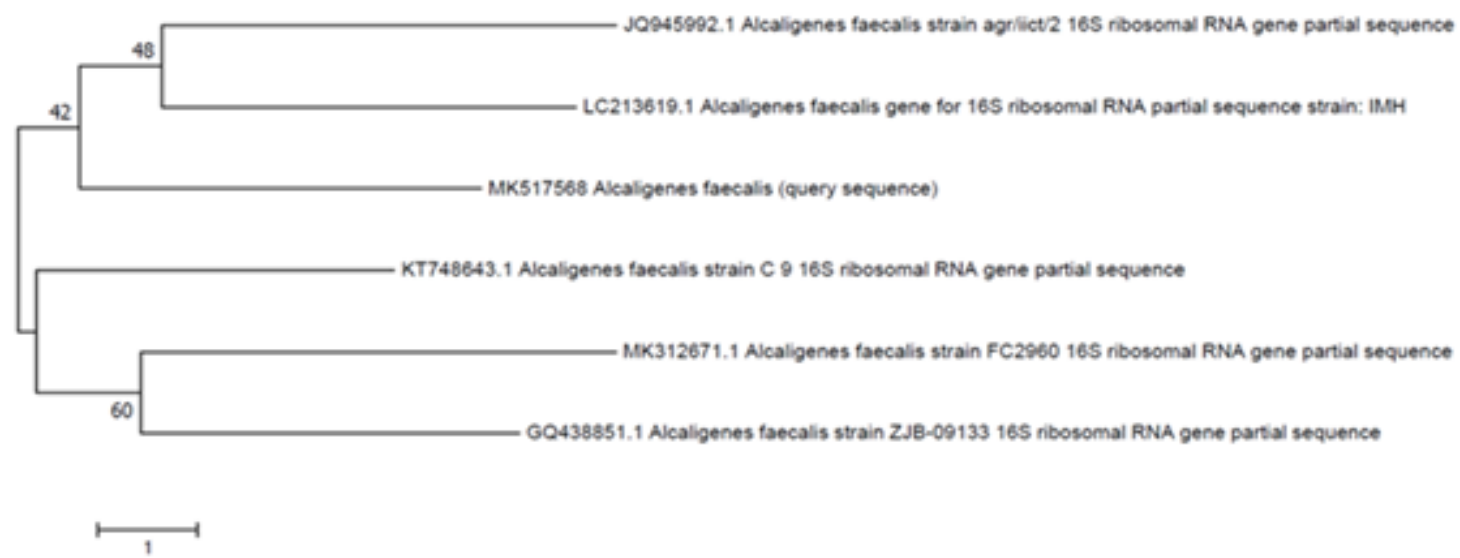

Figure 3

Neighbor joining tree of Alcaligenes faecalis (MK517568)
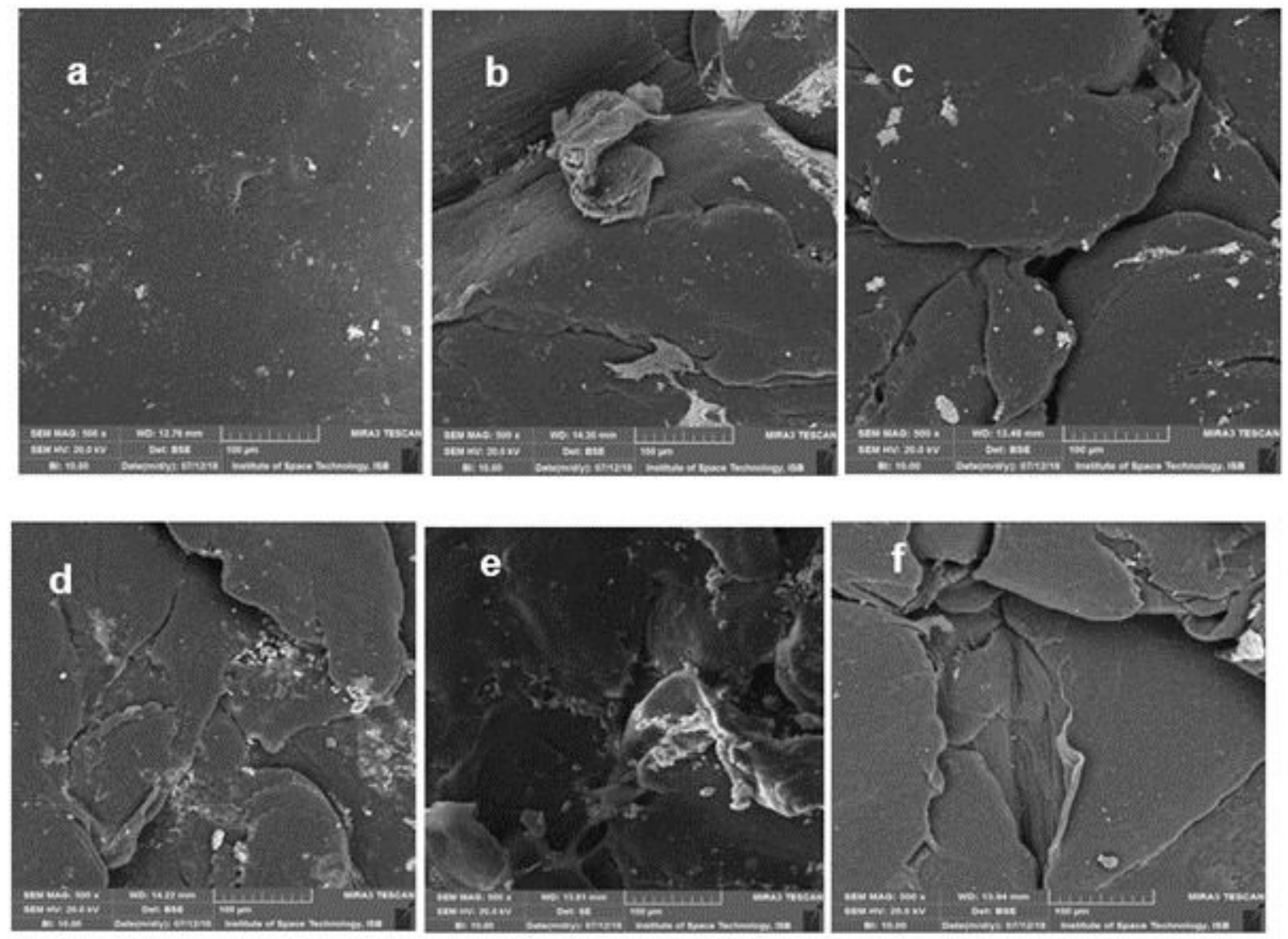

\section{Figure 4}

SEM captures of LLDPE beads following 40 days of incubation by isolates. a) control, b) SA-5 Alcaligenes faecalis treated LLDPE showing bacterial attachment, c) SA-68 Bacillus cereus treated LLDPE showing surface cracks, d) SB-14 treated LLDPE showing surface erosion, e) SC-56 treated LLDPE showing granules of bacterial action, f) SC-9 treated LLDPE showing pith and groove. 

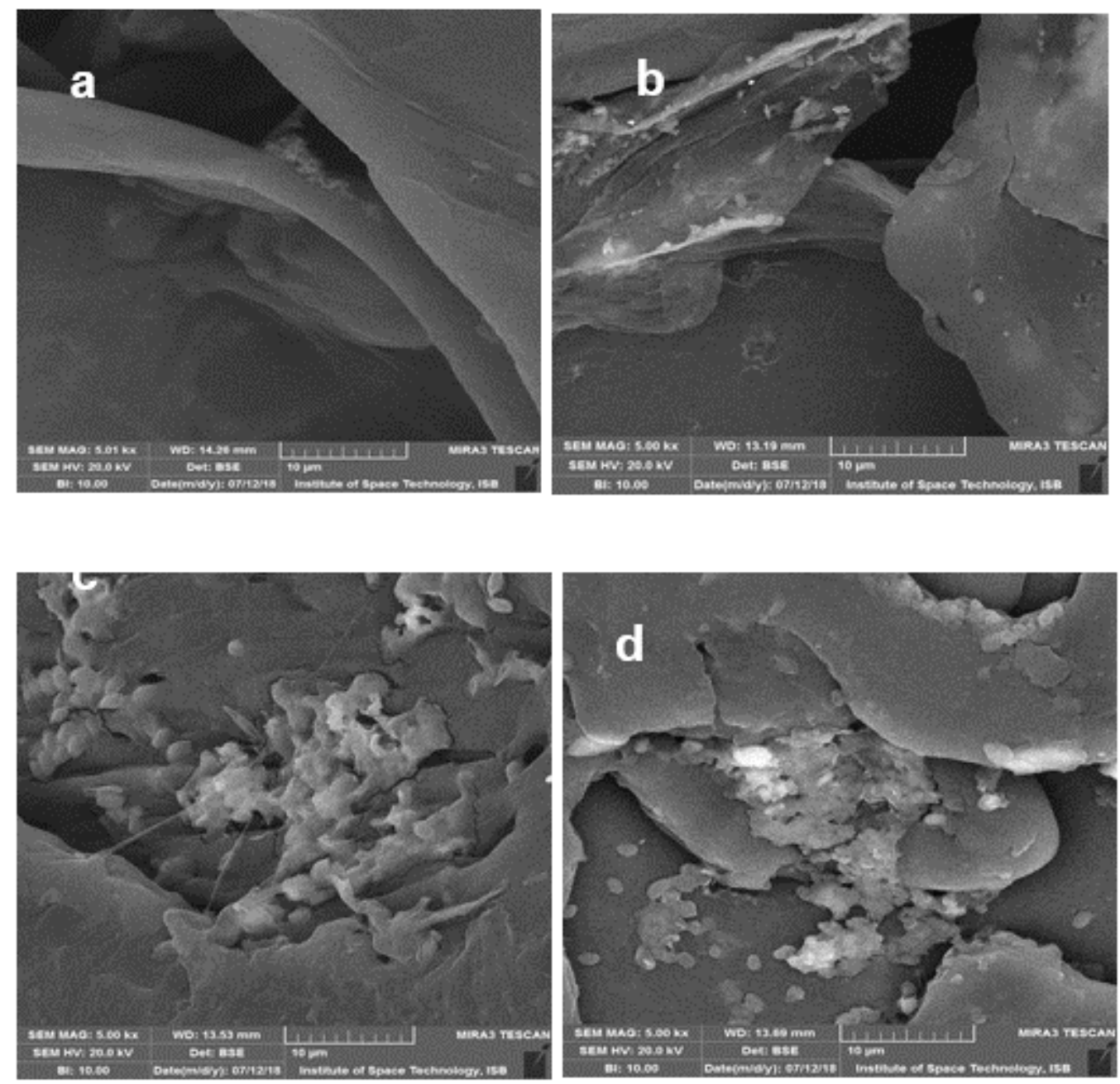

\section{Figure 5}

SEM captures of HDPE beads following 40 days of incubation by isolates. a) control showing no bacteria present in it, b) SA-5 Alcaligenes treated HDPE showing groove, c SB-14 treated HDPE showing bacterial granules, d SC-56 treated HDPE showing surface cracks and bacterial attachment
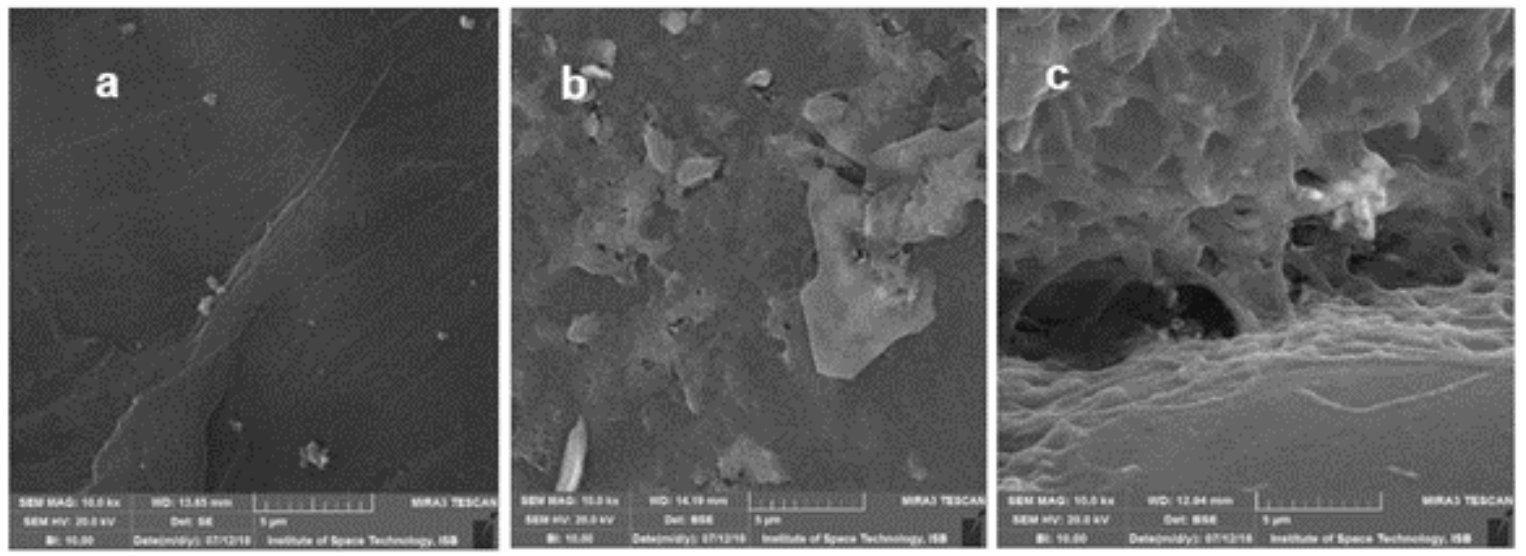

\section{Figure 6}


SEM captures of polyester beads following 40 days of incubation by isolates. a control-smooth surface, $b$ SA-5 Alcaligenes treated polyester showing piths, c SB-14 treated polyester showing groove, piths and holes

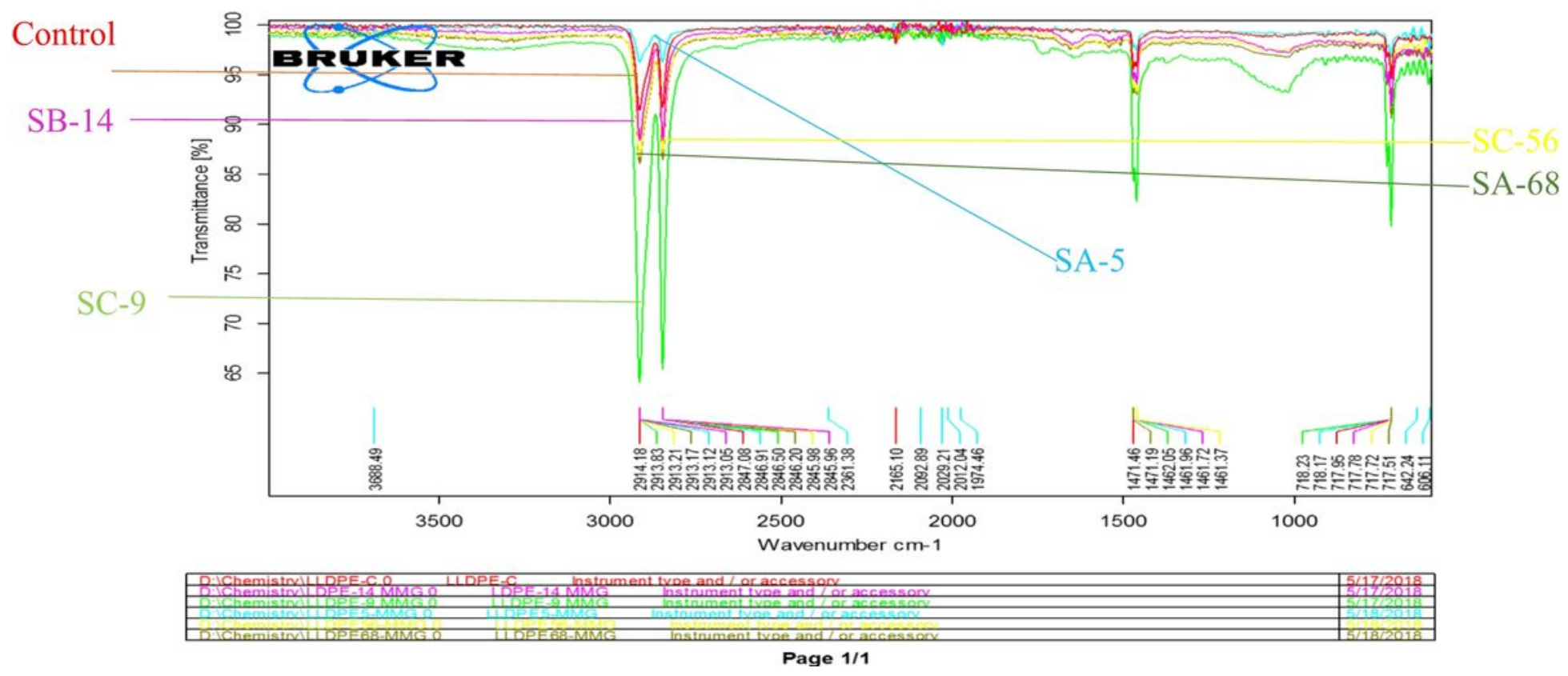

\section{Figure 7}

FT-IR analysis of chemical structural changes of biodegraded LLDPE beads by isolated bacterial strains.

\section{Supplementary Files}

This is a list of supplementary files associated with this preprint. Click to download.

- Supplementarymaterial.docx 